\title{
Beneficios de la Notación de Peirce para los Conectivos Proposicionales Binarios
}

\author{
Benefits of Peirce's notation for propositional binary connectives
}

\section{Benefícios da Notação de Peirce para os Conectivos Proposicionais Binários}

\author{
Oscar Abel Cardona-Hurtado ${ }^{1}$
}

Forma de citar: O. A. Cardona-Hurtado, "Beneficios de la Notación de Peirce para los Conectivos Proposicionales Binarios", Respuestas, vol. 21, no. 1, pp. 56-64, 2016.

Recibido:

Marzo 23 de 2015

Aceptado:

Agosto 27 de 2015

\section{Resumen}

Antecedentes: En la notación tradicional para los conectivos proposicionales binarios son tenidos en cuenta solamente algunos de estos. A lo largo del siglo XX fueron propuestas varias notaciones que subsanan esa falencia, dando lugar al planteamiento de interesantes problemas matemáticos. Objetivo: En este escrito se presenta la notación creada por el norteamericano Charles Peirce, se muestran algunas propiedades de las cuales goza esta simbología, y se evidencian sus ventajas con respecto a la tradicional. Método: Se describe la notación propuesta por Peirce, y se verifican algunas propiedades de carácter lógico geométrico y algebraico entre sus conectivos; también se analiza la posible actuación de estas propiedades en la notación usual. Resultados: Además de varias propiedades individuales y de múltiples relaciones entre los conectivos, las simetrías del sistema completo de los conectivos proposicionales binarios se evidencian de manera visual en los signos propuestos por Peirce. Conclusión: Diversas bondades de las cuales goza la notación propuesta por Peirce, permiten afirmar que la notación usual es superada de manera clara por la simbología diseñada por el científico norteamericano.

Palabras clave: Conectivo proposicional, Charles S. Peirce, operación, simetría, tabla de verdad.

\begin{abstract}
Background: In traditional binary notation for propositional connectives only some of these ones are taken into account. Throughout the twentieth century several notations were proposed which overcome this flaw, leading to the proposal of interesting mathematical problems. Objective: This paper presents the notation created by the American Charles Peirce, showing some of the properties of this symbols, and evidencing the advantages of these compared to the traditional. Method: the notation proposed by Peirce is described, and some properties of the geometric and algebraic logical character among its connective are verified; also, the possible role of these properties in the traditional notation is analyzed. Results: In addition to several individual properties and multiple relations between the connectives, the symmetries of the full set of binary propositional connective is visually evident in the signs proposed by Peirce. Conclusion: Different benefits of the notation proposed by Peirce, support the conclusion that the usual notation is clearly surpassed by the symbolism designed by the American scientist.
\end{abstract}

Keywords: Propositional connective, Charles S. Peirce, operation, symmetry, truth table. 


\section{Resumo}

Antecedentes: Na notação tradicional para os conectivos proposicionais binários são tidos em conta somente alguns destes. Ao longo do século XX foram propostas várias notações que corrigem essa falência, dando lugar ao planejamento de interessantes problemas matemáticos. Objetivo: Neste artigo se apresenta a notação criada pelo norte-americano Charles Peirce, onde se mostram algumas das propriedades que tem está simbologia, e se evidenciam suas vantagens com respeito à tradicional. Método: Se descreve a notação proposta por Peirce, e se verificam algumas propriedades de carácter lógico geométrico e algébrico entre seus conectivos; também se analisa a possível atuação destas propriedades na notação usual. Resultados: Ademais de várias propriedades individuais e de múltiplas relações entre os conectivos, as simetrias do sistema completo dos conectivos proposicionais binários se evidenciam de maneira visual nos signos propostos por Peirce. Conclusão: Diversas bondades se percebem na notação proposta por Peirce, permitindo afirmar que a notação usual é superada de maneira clara pela simbologia desenhada pelo científico norte-americano.

Palavras-chave: Charles S. Peirce, conectivo proposicional, operação, simetria, tabela de verdade.

\section{Introducción}

El cálculo proposicional tradicional se constituye a partir de proposiciones y conectivos lógicos, los segundos sirven de nexo entre las primeras generando así expresiones compuestas. En las presentaciones usuales se tienen en cuenta la negación, la conjunción, la disyunción, la implicación y la equivalencia. Dado que en la lógica clásica solo se consideran dos valores de verdad, estos conectivos pueden ser definidos mediante las llamadas tablas de verdad que se muestran en las tablas I y II, donde el símbolo $\sim$ denota la negación, $A$ y $B$ son proposiciones.

Tabla I. Tabla de verdad de la negación

\begin{tabular}{|c|c|}
\hline $\boldsymbol{A}$ & $\sim \boldsymbol{A}$ \\
\hline$V$ & $F$ \\
$F$ & $V$ \\
\hline
\end{tabular}

Fuente: Autor
Tabla II. Tablas de verdad conectivos binarios notación tradicional

\begin{tabular}{|ll|llll|}
\hline $\boldsymbol{A}$ & $\boldsymbol{B}$ & $\wedge$ & $\vee$ & $\rightarrow$ & $\leftrightarrow$ \\
\hline$V$ & $V$ & $V$ & $V$ & $V$ & $V$ \\
$V$ & $F$ & $F$ & $V$ & $F$ & $F$ \\
$F$ & $V$ & $F$ & $V$ & $V$ & $F$ \\
$F$ & $F$ & $F$ & $F$ & $V$ & $V$ \\
\hline
\end{tabular}

Fuente: Autor

Puesto que la tabla de verdad de cada conectivo consta de cuatro renglones, cada uno de los cuales puede ser $V$ o bien $F$, es fácil ver que en la lógica clásica existen 16 conectivos binarios. Sin embargo, en las presentaciones tradicionales son considerados solamente $4 \mathrm{o}$ 5 de estos. Quizás en los estudios comunes se ha desechado la mayoría de ellos porque cada uno de los que se abordan puede obtenerse como combinación de unos pocos de ellos, como la conjunción y la negación [1], [2], [3], [4]. A lo largo del siglo XX fueron propuestos diversos sistemas de signos que incluyen los 16 conectivos binarios, como es el caso de la notación creada por Charles S. Peirce, según sus escritos elaborada en 1902, y la 
No. 1

Enero - Junio 2016 ISSN 0122-820X E-ISSN 2422-5053 PP: 56-64 formalizada por Shea Zellweger en 1962 [4], [5]. Desafortunadamente estas notaciones son casi desconocidas [3], [4].

El científico, lógico y filósofo norteamericano Charles Sanders Peirce, quien vivió entre 1839 y 1914, y que ha sido reconocido como una de las mentes más brillantes de toda América [6], propuso una notación para los conectivos proposicionales binarios que se puede considerar por muchos motivos, algunos de los cuales se mostrarán de manera explícita en secciones posteriores, una de las más importantes simbologías. En primer lugar, porque son tenidos en cuenta los 16 conectivos existentes: en la notación tradicional, al negar un conectivo se obtiene una tabla de verdad cuyo conectivo no tiene signo. Por ejemplo, la negación de la implicación carece de símbolo propio. El hecho de que en una notación sean tenidos en cuenta los 16 conectivos da lugar a interesantes problemas matemáticos que ni siquiera se pueden plantear en la notación usual. Por otro lado, la notación propuesta por Peirce se destaca por la simetría e iconicidad de sus símbolos. Esto significa que diferentes operaciones lógicas se traducen en movimientos y características de los símbolos empleados, y que a partir de cada uno de estos últimos, se puede construir la tabla de verdad del conectivo que representa.

Desafortunadamente, la simbología propuesta por Charles Peirce es muy poco conocida por la comunidad académica a pesar de tener un gran potencial por explotar [3], [4], [6], [7], [8], [9], [10], [11], [12], [13].
Los aportes al cálculo proposicional de la notación para los conectivos binarios diseñada por el científico norteamericano son significativos: usualmente son estudiados $4 \mathrm{o}$ 5 conectivos, dejando de lado la mayoría de estos. Peirce propuso un sistema homogéneo de signos para los 16 conectivos existentes: esta simbología es icónica, en tanto que los signos contienen el significado de los conectivos y exhiben algunas propiedades de estos; asimismo, los signos reflejan las simetrías del sistema completo de conectivos, lo cual no es posible apreciar con la notación tradicional.

El objetivo de este escrito es presentar la notación para los conectivos binarios propuesta por el norteamericano Charles S. Peirce y resaltar algunas propiedades lógicas, geométricas y algebraicas que se satisfacen en ella, y que en contraste, no sería posible desarrollar en la notación usual. De esta manera, se espera hacer una contribución para que la notación en mención sea cada vez conocida por más personas y el gran potencial que posee sea explotado.

\section{Materiales y métodos}

\subsection{Construcción de la notación propuesta por Charles Peirce}

La idea de Peirce para la construcción de la notación es sencilla, consiste en dos pasos. En primer lugar, se considera el signo $\mathrm{X}$ que tiene cuatro cuadrantes que se rotulan como se muestra en la figura 1.

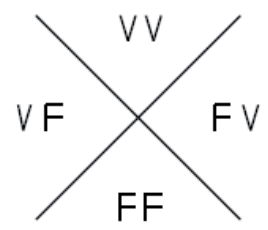

Figura 1. Marco X con cuadrantes rotulados Fuente: Autor

Nótese que la combinación $V V$ es asignada al cuadrante superior, la combinación $F F$ al cuadrante inferior y las combinaciones $V F$ y $F V$ a los cuadrantes izquierdo y derecho respectivamente. Las cuatro parejas de letras $V$ y $F$ relacionadas anteriormente, corresponden a las posibles combinaciones de valores de verdad de dos proposiciones.

El segundo paso consiste en asociar a cada conectivo un dibujo, cerrando al marco $\mathrm{X}$ cada cuadrante para el cual la combinación de valores de verdad sea falsa, y dejando abierto aquel cuya combinación sea verdadera. 
Por ejemplo, el conectivo correspondiente a la implicación es falso únicamente para la combinación $V F$, luego, se le asocia el diagrama presentado en la figura 2 .

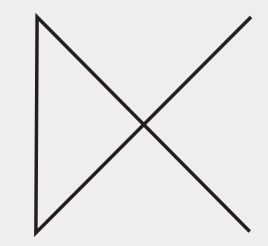

Figura 2. Diagrama asociado a la implicación Fuente: Autor
De esta manera se obtienen 16 dibujos diferentes de figuras $\mathrm{X}$ con cuadrantes que pueden ser abiertos o cerrados según los valores de verdad de los conectivos, como se muestra en la tabla III.

Tabla III. Notación de peirce para los conectivos binarios

\begin{tabular}{|c|c|c|c|c|c|c|c|c|c|c|c|c|c|c|c|c|}
\hline$A B$ & 1 & 2 & 3 & 4 & 5 & 6 & 7 & 8 & 9 & 10 & 11 & 12 & 13 & 14 & 15 & 16 \\
\hline$V V$ & $F$ & $F$ & $F$ & $F$ & $V$ & $V$ & $V$ & $V$ & $F$ & $F$ & $F$ & & $V$ & $V$ & $V$ & $V$ \\
\hline$V F$ & $F$ & $F$ & $F$ & $V$ & $F$ & $V$ & $F$ & $F$ & V & $V$ & $F$ & & $F$ & $V$ & $V$ & $V$ \\
\hline$F \quad V$ & $F$ & $F$ & $V$ & $F$ & $F$ & $F$ & $V$ & $F$ & $V$ & 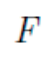 & $V$ & & $V$ & $F$ & $V$ & $V$ \\
\hline \multirow[t]{2}{*}{$F F$} & $F$ & $V$ & $F$ & $F$ & $F$ & $F$ & $F$ & $V$ & $F$ & I & $V$ & $V$ & $V$ & $V$ & $F$ & $V$ \\
\hline & & & & & & & & & & & & & & & & \\
\hline
\end{tabular}

Fuente: Autor

En la última fila de la tabla anterior se encuentran relacionados 16 diagramas distintos, asociados al mismo número de conectivos proposicionales binarios que existen. A cada conectivo le corresponde un único diagrama.

\section{Resultados y análisis}

\subsection{Algunas propiedades de la notación de Peirce}

La notación creada por Peirce presenta interesantes propiedades que se relacionan a continuación.

\subsection{Notación completa}

A diferencia de la notación tradicional, en la cual se estudian 4 o 5 conectivos, en la notación propuesta por Charles Peirce son tenidos en cuenta los 16 conectivos binarios existentes. La importancia de este hecho se verá reflejada en las secciones posteriores.

\subsection{Iconicidad}

En esta notación, los cuadrantes de los diagramas se cierran o no dependiendo del valor de verdad de cada combinación. Al invertir este proceso, el valor de verdad de cada combinación puede determinarse observando el diagrama. Es decir, los diagramas se convierten en íconos de los conectivos, debido a que permiten construir sus tablas de verdad. En la teoría general de los signos propuesta por Peirce, esta característica se conoce como iconicidad. Esto implica que la tabla de verdad de cada conectivo se construye de manera lógica, no es necesario memorizarla como ocurre en la notación tradicional. El signo mismo indica el valor de verdad correspondiente a cada combinación. Por ejemplo, para el conectivo correspondiente a la columna rotulada con el 
número 8 en la tabla III, si las proposiciones $A$ y $B$ tienen diferente valor de verdad, las combinaciones $V F$ y $F V$ correspondientes a los cuadrantes derecho e izquierdo están cerrados, luego estas combinaciones son falsas; las combinaciones $V V$ y $F F$ son verdaderas debido a que los cuadrantes superior e inferior permanecen abiertos.
En los ejemplos siguientes se elaboran las tablas de verdad correspondientes a dos fórmulas, una con dos letras y otra con tres letras. Las tablas IV y V fueron construidas recurriendo a un sencillo ejercicio lógico, partiendo de la forma de los diagramas que representan los conectivos; no fue necesario memorizar las tablas de verdad de estos.

Tabla IV. Tabla de verdad de una fórmula con dos letras

\begin{tabular}{|c|c|c|c|}
\hline$A$ & $B$ & $B \otimes A$ & $A$ 邓 $(B \otimes A)$ \\
\hline \hline$V$ & $V$ & $F$ & $V$ \\
$V$ & $F$ & $F$ & $V$ \\
$F$ & $V$ & $F$ & $V$ \\
$F$ & $F$ & $F$ & $V$ \\
\hline
\end{tabular}

Fuente: Autor

Tabla V. Tabla de verdad de una fórmula con tres letras

\begin{tabular}{|c|c|c|c|c|c|c|}
\hline$A$ & $E$ & $D$ & $A \ltimes D$ & $\sim(A \ltimes D)$ & $E$ 邓 & $\sim(A \ltimes D)$ \ $(E$ 邓 $A)$ \\
\hline \hline$V$ & $V$ & $V$ & $V$ & $F$ & $F$ & $F$ \\
$V$ & $V$ & $F$ & $F$ & $V$ & $F$ & $V$ \\
$V$ & $F$ & $V$ & $V$ & $F$ & $F$ & $F$ \\
$V$ & $F$ & $F$ & $F$ & $V$ & $F$ & $V$ \\
$F$ & $V$ & $V$ & $V$ & $F$ & $V$ & $V$ \\
$F$ & $V$ & $F$ & $V$ & $F$ & $V$ & $V$ \\
$F$ & $F$ & $V$ & $V$ & $F$ & $V$ & $V$ \\
$F$ & $F$ & $F$ & $V$ & $F$ & $V$ & $V$ \\
\hline
\end{tabular}

Fuente: Autor

Así como la notación de Peirce permite resolver tablas de verdad sin necesidad de saber de memoria los valores de verdad de los conectivos, de la misma manera, con esta simbología se pueden demostrar argumentos y equivalencias.

A continuación se presentan dos ejemplos. Primero uno correspondiente a un argumento y luego uno relacionado con una equivalencia.

De $A, A \ltimes B$ se puede deducir $B$, como se muestra en la figura 3 .

\section{$(A \bowtie(A \ltimes B)) \ltimes B$}

Figura 3. Argumento con signos de Peirce Fuente: Autor

La fórmula anterior corresponde a la conocida regla de inferencia Modus Ponens, que puede verificarse resolviendo la tabla de verdad que determina una tautología.
Los signos propuestos por Charles Peirce permiten construir dicha tabla de verdad de manera lógica, a partir de la forma icónica de estos. Por el contrario, al utilizar la notación tradicional, es necesario memorizar la tabla de verdad de cada conectivo.

De otro lado, la equivalencia presentada en la figura 4 corresponde a una ley de De Morgan, reemplazando los signos de los conectivos de Peirce por los de la notación tradicional.

$$
\begin{gathered}
\sim(A \bowtie B) \bowtie(\sim A \searrow \sim B) \\
\text { Figura 4. Equivalencia con signos de Peirce } \\
\text { Fuente: Autor }
\end{gathered}
$$

Evidentemente, la notación diseñada por Peirce es una valiosa herramienta para verificar cualquier equivalencia o regla de inferencia, solucionando la respectiva tabla de verdad. 


\section{4. Álgebra}

Con el surgimiento del sistema de numeración árabe y sus ventajas como la eficacia para realizar de manera rápida operaciones aritméticas, su facilidad para la lectura y escritura, entre otras, este relegó a un segundo plano el sistema de números romanos. Desde entonces estos últimos solo son utilizados para labores decorativas como enumerar los siglos, los actos y las escenas de una obra de teatro, los capítulos de una obra escrita, o los nombres de ciertos personajes como reyes, emperadores y papas. Según Shea Zellweger, con una notación que considere los 16 conectivos existentes pasará algo similar a lo que ocurrió en su momento con el sistema de números árabes; es decir, las bondades de tipo lógico, geométrico y algebraico propias de una notación completa, le permitirán, en algún momento, desplazar a la tradicional [7], [8], [14].

Con los números enteros son estudiadas 4 operaciones básicas: adición $(+)$, sustracción
$(-)$, multiplicación $(\times)$ y división $(\div)$. De manera similar, Shea Zellweger el creador de la notación para los conectivos binarios denominada Alfabeto Lógico, propuso las operaciones lógicas $R 1, R 2, R 3$ y $R 4$. A continuación se definen las cuatro operaciones mencionadas. Aquí * es un conectivo binario cualquiera que relaciona dos proposiciones $A$ y $B$, mientras $\sim$ denota la negación.

- $R 1\left(A^{\star} B\right)=\sim A^{\star} B$

- $R 2\left(A^{\star} B\right)=A\left(\sim^{\star}\right) B$

- $R 3\left(A^{\star} B\right)=A^{*} \sim B$

- $R 4\left(A^{\star} B\right)=B^{\star} A$

En los dos ejemplos siguientes, se verá cómo actúan las operaciones $R l$ y $R 4$ sobre los signos que representan los conectivos binarios.

En efecto, en la figura 5 se muestra cómo actúa R1 sobre el conectivo que se encuentra en la columna rotulada con el número 2 , de la tabla III.

\begin{tabular}{|c|c|c|c|c|c|c|c|c|}
\hline$A$ 冈 & $B$ & \multirow{5}{*}{$\stackrel{R 1}{\rightarrow}$} & $\sim A$ & 冈 & B & & 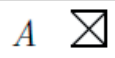 & $B$ \\
\hline$V \quad F$ & V & & & $F$ & V & & $V \quad F$ & V \\
\hline$V \quad F$ & $F$ & & $F$ & $V$ & $F$ & $=$ & $V \quad V$ & $F$ \\
\hline$F \quad F$ & $V$ & & V & $F$ & V & & $F \quad F$ & V \\
\hline$F \quad V$ & $F$ & & V & $F$ & $F$ & & $F \quad F$ & $F$ \\
\hline
\end{tabular}

Figura 5. Efecto de la operación $R l$ sobre un conectivo Fuente: Autor

Se observa que $R l(2)=4$, donde 2 y 4 representan los diagramas que se encuentran en las columnas de la tabla III, rotuladas con los números 2 y 4 respectivamente.
Ahora, la figura 6 muestra cómo actúa $R 4$ sobre el conectivo ubicado en la columna rotulada con el número 8 en la tabla III.

\begin{tabular}{|c|c|c|c|c|c|c|c|c|c|c|}
\hline$A$ & $\bowtie$ & $B$ & \multirow{5}{*}{$\stackrel{R 4}{\rightarrow}$} & & $\bowtie$ & $A$ & & & $\bowtie$ & \\
\hline$V$ & $V$ & $V$ & & & $V$ & $V$ & & $V$ & $V$ & 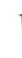 \\
\hline$V$ & $F$ & $F$ & & $F$ & $F$ & $V$ & $=$ & $V$ & $F$ & $I$ \\
\hline$F$ & $F$ & $V$ & & $V$ & $F$ & $F$ & & $F$ & $F$ & \\
\hline$F$ & $V$ & $F$ & & $F$ & $V$ & $F$ & & $F$ & $V$ & \\
\hline
\end{tabular}

Figura 6. Efecto de la operación $R 4$ sobre un conectivo Fuente: Autor 
Vol. 21

No. 1

Enero - Junio 2016 ISSN 0122-820X E-ISSN 2422-5053 PP: 56-64
Se puede apreciar que $R 4(8)=8$; es decir, esta operación aplicada al conectivo de la columna 8 , da como resultado este mismo.

Se puede apreciar que al aplicar cualquiera de las cuatro operaciones a una fórmula $A^{*} B$, de nuevo se obtiene la tabla de verdad de alguno de los 16 conectivos binarios. Esto no ocurre en la notación usual, por ejemplo, al negar la fórmula $A \wedge B$ se produce una tabla de verdad para la cual no existe un conectivo en dicho sistema.

\subsection{Simetrías}

En los ejemplos presentados anteriormente, es posible observar que las operaciones $R l$, $R 2, R 3$ y $R 4$ inducen ciertos movimientos en los signos.

Para precisar el efecto de las operaciones sobre estas figuras se vuelve al marco $\mathrm{X}$ en el plano, donde se conviene que la diagonal ascendente es el segmento creciente de la $\mathrm{X}$, la diagonal descendente es el segmento decreciente. Mientras que el eje horizontal es un segmento que corta el marco $\mathrm{X}$ en su vértice, $\mathrm{y}$ es paralelo algún otro que cierre un cuadrante izquierdo o derecho; asimismo, el eje vertical es un segmento que corta al marco $\mathrm{X}$ en su vértice, $\mathrm{y}$ es paralelo a algún otro que cierra un cuadrante superior o inferior.
Es fácil observar que el efecto de las operaciones sobre los diagramas que representan los conectivos es el siguiente.

- Rl equivale a una reflexión sobre la diagonal ascendente

- $\quad R 2$ corresponde al "complemento" con relación a los cuadrantes cerrados o abiertos

- $R 3$ equivale a una reflexión sobre la diagonal descendente

- $R 4$ corresponde a una reflexión sobre el eje vertical

La combinación de las cuatro operaciones, o movimientos, produce un total de 16 operaciones diferentes. En la tabla VI, que se presenta a continuación, se muestran las 16. En la última columna se relacionan los movimientos que resultan. Las combinaciones de operaciones se efectúan de izquierda a derecha; por ejemplo RIR3 significa que primero se realiza la operación $R 1$ y luego $R 3$. La letra $I$ denota la operación idéntica. Por otro lado, las abreviaturas Re, Ro, Co, $\mathrm{EV}, \mathrm{EH}, \mathrm{DA}$ y DD indican, en ese orden: reflexión, rotación, complemento, eje vertical, eje horizontal, diagonal ascendente y diagonal descendente.

Tabla VI. Efecto de las operaciones sobre los signos

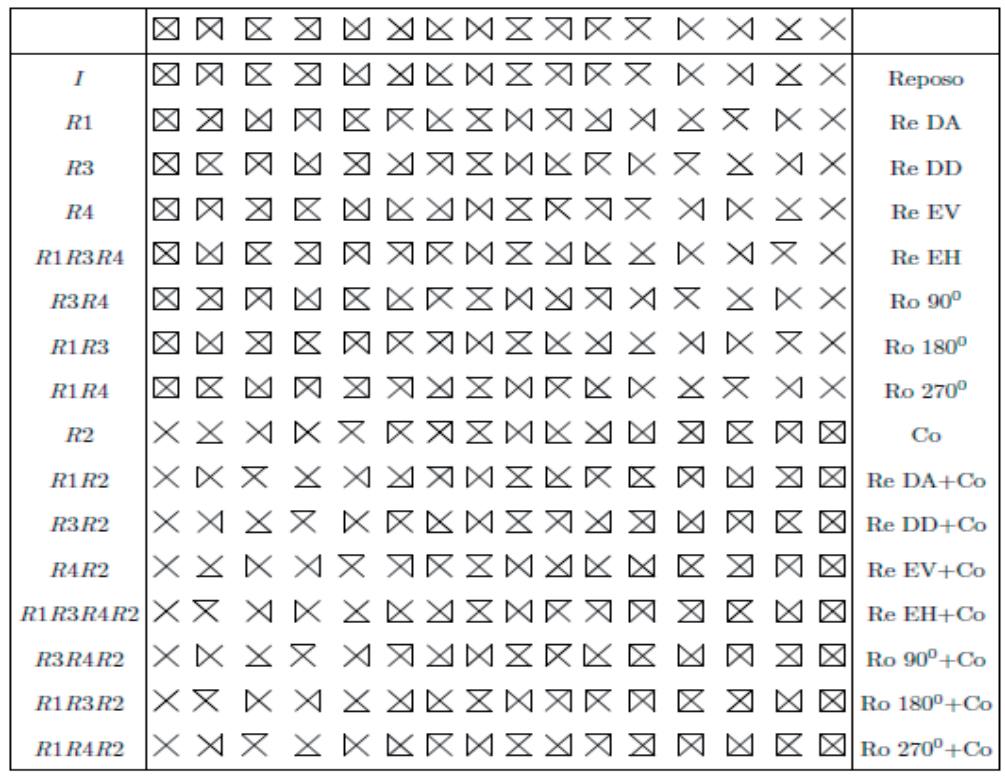


La tabla VI presenta algunas características notables, entre otras: en cada fila aparecen los 16 signos y ninguno se repite; en cada una de las 8 primeras filas el número de cuadrantes va disminuyendo; en cada una de las 8 últimas filas el número de cuadrantes cerrados va en aumento; los 16 signos de cada fila pueden agruparse en subconjuntos preservando el orden 14641 según el número de cuadrantes cerrados o abiertos; también se destaca que las últimas 8 filas son el complemento de las 8 primeras.

Puede demostrarse técnicamente que la tabla VI constituye un grupo de 16 elementos [3], [4], [8], [15]. Las simetrías del sistema de los conectivos binarios de la lógica clásica están dadas por los invariantes [16] de este grupo, y son reveladas en la simbología propuesta por Peirce en la anterior tabla.

\section{Conclusiones}

La notación para los conectivos proposicionales binarios propuesta por Charles Sanders Peirce puede considerarse como un modelo de signos completo y diagramático, pues son considerados los 16 conectivos existentes, además cada signo está relacionado con un diagrama que permite construir el objeto que representa. Los signos revelan las propiedades de los conectivos y las relaciones entre estos; el conjunto de signos evidencia las propiedades del sistema de conectivos. Esta simbología creada por Peirce no admite comparación con la notación tradicional, la supera ampliamente.

\section{Referencias}

[1] X. Caicedo. Elementos de Lógica y Calculabilidad. Bogotá, una Empresa Docente, 1990.

[2] O. A. Cardona. Álgebra en el Alfabeto Lógico de Zellweger. Tesis de Especialización en Matemáticas, Universidad del Tolima, Ibagué, 2011.

[3] M. García, J. F. Gómez. Notación de Peirce para los conectivos binarios.
Tesis de Matemáticas, Universidad del Tolima, Ibagué, 2002.

[4] A. Oostra. "La notación diagramática de Peirce para los conectivos proposicionales binarios", Revista de la Academia Colombiana de Ciencias, vol. 28, no. 106, pp. 57-70, 2004.

[5] L. Granados, N. Aya. "Geometría en el Alfabeto Lógico de Zellweger", Memorias del XX Encuentro de Geometría y sus Aplicaciones, (Bogotá), pp. 361-369, Universidad Pedagógica Nacional, 2011.

[6] A. Oostra. "Una reseña de la lógica matemática de Charles S. Peirce", REVISTA Universidad EAFIT, vol. 44, no. 150, pp. 9-20, 2008.

[7] G. Clark, S. Zellweger. "Let the mirrors on the thinking", Mount Union Magazine, vol. 93, pp. 113-120, 1993.

[8] S. Zellweger. "Untapped potential in Peirce's iconic notation for the sixteen binary connectives" Studies in the Logic of Charles Peirce, pp. 334-386, 1997.

[9] P. Farias, J. Queiroz. "Nuevas estrategias para modelar procesos y estructuras sígnicas", SIGNA revista de Asociación Española de Semiótica, vol. 1, no. 10, pp. 128-148, 2001.

[10] A. Oostra. "Acercamiento lógico a Peirce", Boletín de Matemáticas, vol. 7, no. 2, pp. 60-77, 2000.

\begin{tabular}{l}
1 \\
$22-820 X$ \\
$622-5053$ \\
$6-64$ \\
\hline
\end{tabular}

Cúcuta-Colombia 
Vol. 21

No. 1

Enero - Junio 2016

ISSN 0122-820X

E-ISSN 2422-5053

PP: 56-64

[14] A. Oostra. "Simetría en algunas tablas de C.S. Peirce", Memorias del Encuentro de Geometría y sus Aplicaciones, (Bogotá), pp. 1-49, Universidad Pedagógica Nacional, 2003.

[15] M. García, J. F. Gómez, A. Oostra. "Simetría y Lógica: la notación de Peirce para los 16 conectivos binarios", Memorias del XII Encuentro de Geometría y sus Aplicaciones, (Bogotá), pp. 1-26, Universidad Pedagógica Nacional, 2001.

[16] H. Weyl. La Simetría. MacGraw-Hill, Madrid, 1990. 